\title{
Rational Points on Some Fano Quadratic Bundles
}

\author{
Andreas-Stephan Elsenhans
}

\section{CONTENTS}

1. Introduction

2. The Tamagawa Number

3. Computation of the Tamagawa Number

4. Some Subvarieties of the Threefold

5. Counting Rational Points

6. Discussion

Acknowledgments

References

2000 AMS Subject Classification: 11D45, 11Y50, 14G05

Keywords: quadratic bundle, Manin's Conjecture, accumulating subvariety
We study the number of rational points of bounded height on a certain threefold. The accumulating subvarieties are Zariski dense in this example. The computations support an extension of a conjecture of Manin to this situation.

\section{INTRODUCTION}

The set of rational points on varieties is one of the central objects in arithmetic geometry. For Fano varieties, many rational points are expected (at least after an extension of the ground field). So one could ask for the number of such points of bounded height. This leads to the famous conjecture of Yuri Manin [Franke et al. 89].

Conjecture 1.1. (Manin.) Let $V$ be an arbitrary Fano variety and $H$ the anticanonical height. Then there exists a dense Zariski-open subset $V^{\circ} \subset V$ such that for each number field $K$,

$$
\#\left\{x \in V^{\circ}(K) \mid H(x)<B\right\} \sim C \cdot B \log ^{r} B,
$$

where $r$ is expected to be $\operatorname{rk} \operatorname{Pic}(V)-1$.

To illustrate the choice of the Zariski-open subset $V^{\circ} \subset V$, we give an example.

Example 1.2. A smooth cubic surface with 27 rational lines has Picard rank 7. So we expect approximately $C$. $B \log ^{6} B$ rational points of height at most $B$. But every line has approximately $C B^{2}$ rational points. In this case, one chooses $V^{\circ}$ as the complement of the lines.

Examples in which the rational points in the exceptional set have the same magnitude as the rational points in $V^{\circ}$ are given in [Elsenhans and Jahnel 06b] and [Hooley 86].

The Manin conjecture is proven in a number of special cases. In the case of high dimension and low degree, this can be done using the circle method [Birch 62]. Beyond this, there are many (theoretical 
and numerical) results supporting this conjecture. On the other hand, we have the following counterexample [Batyrev and Tschinkel 96]:

Example 1.3. Let $V \subset \mathbf{P}^{3} \times \mathbf{P}^{3}$ be the Fano variety

$$
\begin{gathered}
\{([a: b: c: d],[x: y: z: w]) \mid \\
\left.a x^{3}+b y^{3}+c z^{3}+d w^{3}=0\right\} .
\end{gathered}
$$

The number of rational points over $\mathbb{Q}\left(\zeta_{3}\right)$ grows at least as fast as $B \log ^{3} B$ on each nonempty Zariskiopen subset, but the conjecture predicts approximately $C B \log B$.

The point is that the fibers with $a, b, c, d$ cubes are cubic surfaces with Picard rank 7. On these fibers, $C B \log ^{6} B$ rational points are expected. The lower bound $B \log ^{3} B$ is proven. Since these fibers form a Zariskidense subset, we get a counterexample to the Manin conjecture.

At this point at least the following possibilities arise:

- The class of all Fano varieties is too big for a uniform conjecture.

- The value of the constant $r$ has to be modified.

- The requirement of $V^{\circ}$ to be Zariski open is too strong.

Remark 1.4. Aside from this counterexample, several people have extended the conjecture. Most important is a conjectural value of the constant $C$. This is expected to be a Tamagawa-type number introduced by E. Peyre [Peyre 95]. (See below for more details.)

Furthermore, attempts have been made to construct a more precise asymptotic formula for the number of rational points [Swinnerton-Dyer 05]. It is given by $B P(\log B)+O\left(B^{\frac{1}{2}+\varepsilon}\right)$. Here, $P$ is a polynomial of degree $\operatorname{rk} \operatorname{Pic}(V)-1$ with leading coefficient $C$, and $\varepsilon$ is some value in $\left(0, \frac{1}{2}\right)$. See [de la Bretèche et al. 07] for a proof in the case of a special singular cubic surface.

In this note we focus on varieties of the form

$$
a x^{2}+b y^{2}+l_{1}(a, b) z^{2}+l_{2}(a, b) w^{2}=0
$$

in $\mathbf{P}^{1} \times \mathbf{P}^{3}$. Here $l_{1}$ and $l_{2}$ are two linear forms. This example was suggested by Emmanuel Peyre and Yuri Tschinkel during the arithmetic and algebraic geometry conference of higher-dimensional varieties (Bristol 2009). After a study of the geometry of these varieties we will search for rational points and compare their number with the predicted value.

For a numerical check of the Manin conjecture in simpler cases, the reader might consult [Peyre and Tschinkel 01], [Elsenhans and Jahnel 06b], or [Elsenhans and Jahnel 09]. Arguments and computations carried out in great detail there are only sketched in this note.

\section{THE TAMAGAWA NUMBER}

For an arbitrary Fano variety, a conjectural value of $C$ was introduced by E. Peyre. It is an infinite product of Tamagawa type. We will recall this constant in a special case, which is yet general enough for our situation.

More precisely, we will work over $\mathbb{Q}$, and we assume that the Galois action on the Picard group of the variety is trivial. The latter implies that we do not have to care about the Brauer-Manin obstruction. Furthermore, we will restrict our attention to varieties given by a single equation in a product of projective spaces. Then $C$ is given as the following product:

$$
C=\alpha \prod_{p \in \mathbb{P} \cup\{\infty\}} \tau_{p}
$$

For a prime $p \in \mathbb{P}$, the local factor $\tau_{p}$ is given by

$$
\tau_{p}=\left(1-\frac{1}{p}\right)^{\mathrm{rkPic}(V)} \cdot \lim _{k \rightarrow \infty} \frac{\# V\left(\mathbb{Z} / p^{k} \mathbb{Z}\right)}{p^{k \operatorname{dim} V}} .
$$

At the infinite place we have the following formula:

$$
\tau_{\infty}=\frac{(n-d+1)(m-e+1)}{4} \int_{\mathrm{CU} \cap N} \omega_{\text {Leray }}
$$

for a variety in $\mathbf{P}^{n} \times \mathbf{P}^{m}$ given by a polynomial of bidegree $(d, e)$. Here, $\mathrm{CU}$ is the affine cone given by the equation, and $N$ is $[-1,1]^{n+m+2}$. Finally, $\alpha$ is given by

$$
\alpha=\operatorname{rk} \operatorname{Pic}(V) \cdot \operatorname{vol}\left\{x \in \Lambda_{\text {eff }}^{\vee} \mid\langle x \mid-K\rangle \leq 1\right\} .
$$

Here $\Lambda_{\text {eff }}^{\vee}$ is the dual cone of the cone of effective divisors $\Lambda_{\text {eff }}(V) \subset \operatorname{Pic}(V)^{\vee} \otimes \mathbb{R}=\mathbb{R}^{\text {rk } \operatorname{Pic}(V)}$, and vol denotes the Lebesgue measure on $\operatorname{Pic}(V)^{\vee} \otimes \mathbb{R}$, normalized such that the primitive cell of the lattice $\operatorname{Pic}(V)^{\vee}$ is of measure 1 .

\section{COMPUTATION OF THE TAMAGAWA NUMBER}

Now we consider a variety of the form (1-1). Note that the Picard group is isomorphic to $\mathbb{Z}^{2}$. 


\subsection{One Local Factor}

At a place of good reduction, the local factor is

$$
\tau_{p}=\left(1-\frac{1}{p}\right)^{2} \frac{\# V\left(\mathbb{F}_{p}\right)}{p^{3}}
$$

At a place of bad reduction, the sequence in the definition becomes stationary after a finite number of steps.

At a place of good reduction, $\# V\left(\mathbb{F}_{p}\right)$ can be computed as follows. The variety $V$ is given by the equation $a x^{2}+b y^{2}+l_{1}(a, b) z^{2}+l_{2}(a, b) w^{2}=0$. For a fixed point $[a: b] \in \mathbf{P}^{1}$ we get a quadric in $\mathbf{P}^{3}$. Since we are at a place of good reduction, only the following three cases are possible:

(i) The quadric is smooth and the discriminant is not a square. Then it has $p^{2}+1$ points.

(ii) The quadric is smooth and the discriminant is a square. Then it has $p^{2}+2 p+1$ points.

(iii) The quadric is singular. Exactly one coefficient is zero. In this case, we get $(p+1) p+1$ points. This happens exactly four times.

Summarizing, we get $p^{2}+p+1+\left(\frac{F(a, b)}{p}\right) p$ points on a fiber. Here we set $F(a, b):=a b l_{1}(a, b) l_{2}(a, b)$.

We introduce the elliptic curve

$$
E: u^{2}=F(a, b)
$$

as a double cover of $\mathbf{P}^{1}$. Points on $E$ with $u \neq 0$ correspond to split quadrics. Thus we get the following formula:

$$
\begin{aligned}
\# V\left(\mathbb{F}_{p}\right)= & 4\left(p^{2}+p+1\right)+\left(p^{2}+1\right)(p+1-4) \\
& +\left(\# E\left(\mathbb{F}_{p}\right)-4\right) p \\
= & p^{3}+2 p^{2}+2 p+1-p T_{p}(E) .
\end{aligned}
$$

Here $T_{p}(E)$ denotes the trace of Frobenius on $E$ at $p$. Note that $\left|T_{p}(E)\right|<2 \sqrt{p}$ by Hasse's theorem. This leads to

$$
\begin{aligned}
\tau_{p} & =\left(1-\frac{2}{p}+\frac{1}{p^{2}}\right) \frac{p^{3}+2 p^{2}+2 p+1-p T_{p}(E)}{p^{3}} \\
& =1-\frac{1}{p^{2}}-\frac{1}{p^{3}}+\frac{1}{p^{5}}-T_{p}(E)\left(\frac{1}{p^{2}}-\frac{2}{p^{3}}+\frac{1}{p^{4}}\right) .
\end{aligned}
$$

\subsection{The Infinite Product}

The infinite product of the local factors calculated above is absolutely convergent. The local factors have many similarities with local factors of the $L$-series $L$ of $E$ and the Riemann zeta function $\zeta$. The local factor $L_{p}(s)$ is given by $\left(1-T_{p}(E) p^{-s}+p^{1-2 s}\right)^{-1}$ at places of good reduction, and $\zeta_{p}(s)$ is simply $\left(1-p^{-s}\right)^{-1}$. Thus we have $\zeta(s)=\prod_{p} \zeta_{p}(s)$ and $L(s)=\prod_{p} L_{p}(s)$. Extracting such factors improves the convergence properties. We get

$$
\begin{aligned}
\prod_{p} \tau_{p}= & \frac{L(3)^{2}}{\zeta(2) \zeta(3)^{2} L(2) L(4)^{2}} \\
& \times \prod_{p} \frac{\zeta_{p}(2) \zeta_{p}(3)^{2} L_{p}(2) L_{p}(4)^{2}}{L_{p}(3)^{2}} \tau_{p} .
\end{aligned}
$$

The factors of the new product are $1+\frac{2 T_{p}(E)^{2}}{p^{5}}+$ $O\left(p^{-9 / 2}\right)$.

In this way, we can evaluate the Euler product with a precision of 14 digits within a few minutes. Most of the time is used for the evaluation of the $L$-series. Here we use MAGma. See [Dokchitser 04] for details of the algorithm.

\subsection{The Infinite Place}

The factor at the infinite place is a 5-dimensional integral on a compact domain. Using homogeneity, we can reduce to $a$ or $b$ equal to 1 and $1 \in\{x, y, z, w\}$. This is a sum of eight 3-dimensional integrals. The summand for $a=x=$ 1 is given by

$$
\iiint \frac{1}{\left|y^{2}+l_{1}(0,1) z^{2}+l_{2}(0,1) w^{2}\right|} d y d z d w .
$$

The other seven cases are similar. The domain of integration for the case $a=x=1$ is given by

$$
\left\{(y, z, w) \in[0,1]^{3}|| \frac{1+l_{1}(1,0) z^{2}+l_{2}(1,0) w^{2}}{y^{2}+l_{1}(0,1) z^{2}+l_{2}(0,1) w^{2}} \mid \leq 1\right\} .
$$

The innermost integral can be evaluated by hand. The remaining 2-dimensional integral can be evaluated using standard methods from numerical analysis. Here we apply an adaptive version of the iterated Gauss-Legendre method. We expect a precision of at least six decimal places.

\section{SOME SUBVARIETIES OF THE THREEFOLD}

\subsection{The Quadric Fibration}

First recall the meaning of fibration into quadrics. A fixed point $[a: b] \in \mathbf{P}^{1}$ leads to the quadric $V_{a, b}$ in $\mathbf{P}^{3}$. This quadric is singular if and only if one of $a, b, l_{1}(a, b), l_{2}(a, b)$ is zero.

The quadric is a split quadric (i.e., has Picard rank 2 over $\mathbb{Q}$ ) if and only if its discriminant is a square and it has a rational point.

Thus, a necessary condition for a quadric to be split is that it correspond to a point on the elliptic curve $E$ introduced above by the equation $u^{2}=a b l_{1}(a, b) l_{2}(a, b)$. 
Furthermore, note that the map

$$
[a: b ; u] \mapsto\left(\frac{a}{b}, \frac{l_{1}(a, b)}{b}, \frac{l_{2}(a, b)}{b}\right) \in\left(\mathbb{Q} / \mathbb{Q}^{2}\right)^{3}
$$

for $[a: b ; u] \in E(\mathbb{Q})$ with $u \neq 0$ extends to a homomorphism $\phi: E(\mathbb{Q}) \rightarrow\left(\mathbb{Q}^{*} / \mathbb{Q}^{* 2}\right)^{3}$ with kernel $2 E(\mathbb{Q})$ [Silverman 86, Chapter X, Proposition 1.4].

This shows that if $[a: b ; u]$ leads to a split quadric, than all points in the coset $[a: b ; u]+2 E(\mathbb{Q})$ lead to singular or split quadrics.

If $E$ has positive rank and at least one split quadric exists, then all split quadrics form a Zariski-dense subset of the threefold. We will give examples for this below.

\subsection{An Elliptic Cylinder}

Another interesting subvariety of $V$ is the following. Rewrite the equation as $a q_{1}(x, y, z, w)+b q_{2}(x, y, z, w)=$ 0 . From this we get the subvariety

$$
\begin{gathered}
\mathbf{P}^{1} \times\left\{[x: y: z: w] \in \mathbf{P}^{3} \mid q_{1}(x, y, z, w)\right. \\
\left.=q_{2}(x, y, z, w)=0\right\} .
\end{gathered}
$$

This is the product of $\mathbf{P}^{1}$ and a genus- 1 curve.

One could count the points on $V\left(\mathbb{F}_{p}\right)$ by checking whether $[x: y: z: w] \in \mathbf{P}^{3}$ leads to a point or a line on $V$. This leads to the observation that this genus-1 curve has the same Frobenius trace as the elliptic curve $E$ given above. Further one can check that both curves have the same j-invariant. But it may happen that only one of the curves has a rational point.

\section{COUNTING RATIONAL POINTS}

\subsection{The Point-Counting Algorithm}

Our variety $V$ is given by the equation

$$
a x^{2}+b y^{2}+l_{1}(a, b) z^{2}+l_{2}(a, b) w^{2}=0
$$

in $\mathbf{P}^{1} \times \mathbf{P}^{3}$. Because of symmetry, we restrict to nonnegative values for $x, y, z, w$. Points with one zero are counted with weight $\frac{1}{2}$. Points with two or three zeros are counted with weights $\frac{1}{4}$ and $\frac{1}{8}$.

Recall that the anticanonical height is given by the formula

$$
\begin{aligned}
& H([a: b ; x: y: z: w]) \\
& \quad=H_{\text {naive }}([a: b]) H_{\text {naive }}([x: y: z: w])^{2} .
\end{aligned}
$$

For a point of bounded height, at least one of the factors is small. This observation leads to the following splitting. Choose a search bound $B$ and an auxiliary bound $A$.
For all $[a: b] \in \mathbf{P}^{1}$ with $H_{\text {naive }}([a: b])<A$, search for rational points on the quadric $C_{a, b}$ of naive height at most $\sqrt{B / H([a: b])}$.

For all points $[x: y: z: w] \in \mathbf{P}^{3}$ with

$$
H_{\text {naive }}([x: y: z: w])<\sqrt{\frac{B}{A}},
$$

solve the linear Diophantine equation for $[a: b]$. If this is the zero equation, we get a line on the elliptic cylinder. If it is not the zero equation, we get a point. This point leads to the solution $[a: b ; x: y: z: w]$. We take it if $|a|$, $|b|$ is below $B / H([x: y: z: w])^{2}$.

The optimal value of the auxiliary bound $A$ depends on details of the implementation and the machine. We took $A=400$ for $B=10^{8}$. Searching for points on $C_{a, b}$ is fast and easy. Just apply the ideas of [Elsenhans and Jahnel 06a], [Elsenhans and Jahnel 06b], and [Elsenhans and Jahnel 10]. Some optimizations are possible using congruences.

\subsection{Rational Points on Subvarieties}

Recall that the Manin conjecture is proven for degree-2 surfaces. It leads to $\sim C B$ points of height at most $B$ on nonsplit and $\sim C B \log B$ points on split quadrics.

The singular fibers lead to $\sim C B \log B$ points of anticanonical height at most $B$ on $V$.

Counting points on the elliptic cylinder means counting points on the elliptic curve itself. Since the heights of rational points on elliptic curves grow rapidly, we get $\sim C B^{2}$ rational points on the elliptic cylinder with anticanonical height at most $B$ on $V$.

\subsection{Numerical Results}

To get an overview of the different phenomena, we list all the data for three examples. Choosing examples is always more or less random. Here we take examples with a considerable proportion of points on the split and the singular quadrics:

$$
\begin{aligned}
& V_{1}: a x^{2}+b y^{2}+(a-b) z^{2}+(a-2 b) w^{2}=0, \\
& V_{2}: a x^{2}+b y^{2}+(2 a-b) z^{2}+(-6 a+b) w^{2}=0, \\
& V_{3}: a x^{2}+b y^{2}+(-91 a-92 b) z^{2}+(99 a+100 b) w^{2}=0 .
\end{aligned}
$$

We apply the point-search algorithm described above. Points on the elliptic cylinder were excluded from the count. We counted the points in each height interval of length $10^{5}$ up to $10^{8}$. Some of the values are listed in Tables 1 and 2 .

We approximate the number of rational points by functions of the form $c_{1} B \log (B)+c_{2} B$ using the 


\begin{tabular}{lccr}
\hline Number of points & $V_{1}$ & $V_{2}$ & $V_{3}$ \\
\hline altogether & 593147.75 & 910514 & 313622 \\
on singular quadrics & 262910.75 & 276816 & 111 \\
on split quadrics & 0 & 121182.5 & 263390 \\
on nonsplit quadrics & 330237 & 512515.5 & 50121 \\
\hline
\end{tabular}

TABLE 1. Number of rational points of anticanonical height below $10^{5}$.

least-squares method. The root-mean-square error of the approximations is always below $0.03 \%$. The results are listed in Table 3.

The values of the Tamagawa numbers are listed in Table 4.

\subsection{Conclusion}

We observe a good coincidence of Peyre's constant and the leading coefficient for the approximate formula for the number of rational points outside the elliptic cylinder on smooth nonsplit fibers.

\subsection{The Split Quadrics}

Now we take a closer look at the split quadrics.

The split quadrics for $V_{2}$ are given by

$$
\begin{aligned}
(a, b) \in\{ & (1,-2),(1,-48),(1,-6),(1,3),(1,4), \\
& (4,-1),(9,50),(25,54),(49,-1058),(169,867), \\
& (289,676),(529,-294),(3600,-36481), \\
& (36481,-43200),(43681,116162), \\
& (58081,262086),(8958049,52911076), \\
& (13227769,26874147),(17497489,-837218), \\
& \ldots\}
\end{aligned}
$$

\begin{tabular}{cc}
\hline & Number of points of \\
Set of points & $0.477469 B \log (B)$ \\
& $+0.436195 B$ \\
\hline on $V_{1}$ altogether & $0.312111 B \log (B)$ \\
without singular quadrics & $-0.297472 B$ \\
on nonsplit quadrics & $0.312111 B \log (B)$ \\
& $-0.297472 B$ \\
\hline on $V_{2}$ altogether & $0.925062 B \log (B)$ \\
& $-1.588194 B$ \\
without singular quadrics & $0.742798 B \log (B)$ \\
& $-2.272602 B$ \\
on nonsplit quadrics & $0.652422 B \log (B)$ \\
& $-2.441540 B$ \\
on split quadrics & $0.090376 B \log (B)$ \\
& $+0.168938 B$ \\
\hline on $V_{3}$ altogether & $0.225190 B \log (B)$ \\
& $+0.538876 B$ \\
without singular quadrics & $0.225028 B \log (B)$ \\
& $+0.540088 B$ \\
on nonsplit quadrics & $0.054220 B \log (B)$ \\
& $-0.125521 B$ \\
on split quadrics & $0.170809 B \log (B)$ \\
& $+0.665609 B$ \\
\hline
\end{tabular}

TABLE 3. Experimental formulas for the number of rational points.

The split quadrics for $V_{3}$ are given by

$$
\begin{aligned}
(a, b) \in\{ & (1,-1),(1,-25),(4900,-8019), \\
& (96100,-107811),(101761,-198025), \\
& (261121,-259081),(504100,-527571), \\
& (2989441,-2961841), \ldots\} .
\end{aligned}
$$

Since the coefficients of these quadrics grow rapidly, the density of rational points decreases rapidly. In the search

\begin{tabular}{lccc}
\hline Number of points & $V_{1}$ & $V_{2}$ & $V_{3}$ \\
\hline altogether & 923032815.25 & 1545094531.75 & 468691343.75 \\
on singular quadrics & 377837495.25 & 404003678 & 173805 \\
on split quadrics & 0 & 183375308 & 381163936.5 \\
on nonsplit quadrics & 545195320 & 957715545.75 & 87353602.25 \\
\hline
\end{tabular}

TABLE 2. Number of rational points of anticanonical height below $10^{8}$. 


\begin{tabular}{llll}
\hline & \multicolumn{1}{c}{$V_{1}$} & \multicolumn{1}{c}{$V_{2}$} & \multicolumn{1}{c}{$V_{3}$} \\
\hline$\alpha$ & 0.25 & 0.25 & 0.25 \\
$\tau_{\infty}$ & 2.8331245 & 3.2014086 & 0.34700116 \\
$\prod_{p} \tau_{p}$ & 0.441828484431 & 0.819836740267 & 0.627048795633 \\
$C_{\text {Peyre }}$ & 0.3129388 & 0.6561581 & 0.05439666 \\
\hline
\end{tabular}

TABLE 4. Approximate values of Tamagawa numbers.

range, only the first two split quadrics on $V_{3}$ have rational points.

We calculate $\sum \frac{\tau\left(C_{a, b}\right)}{H_{\text {naive }}([a: b])}$. That is, we compute the sum of the Tamagawa numbers of the split quadrics. The convergence of the series is fast. The smallest contribution of a quadric listed above is of magnitude $10^{-16}$. The sum over these quadrics is very close to the limit. For $V_{2}$ and $V_{3}$ these sums are respectively approximately 0.0903666 and 0.170788 .

\subsection{Conclusion}

We observe a good coincidence of the sum of the Tamagawa numbers of the split quadrics and the leading coefficient of the experimental formula for the number of rational points in these fibers.

\section{DISCUSSION}

The computations show that the Manin conjecture as presented in the introduction does not hold for quadratic bundles, since it allows only the exclusion of Zariskiclosed sets such as the singular fibers and the elliptic cylinder. For this, the smooth split fibers contain too many points, and the predicted number of points is too small.

The examples suggest the following possibilities:

- Modify the formula for the expected number of rational points. That is, add a term for the contribution of the split fibers.

- Allow the exceptional set to be a thin Zariski-dense part of the variety, for example, fibers given by rational points on an elliptic curve.

For a theoretical treatment, compare E. Peyre's talk "Freedom and Goodness," given at the conference Arithmetic and Algebraic Geometry of Higher-Dimensional Varieties, Bristol, September 2009.

\section{ACKNOWLEDGMENTS}

The author was supported by the Deutsche Forschungsgemeinschaft (DFG) through a funded research project.

\section{REFERENCES}

[Batyrev and Tschinkel 96] V. Batyrev and Y. Tschinkel. "Rational Points on Some Fano Cubic Bundles." C. R. Acad. Sci., Ser. I, Paris 323 (1996), 41-46.

[Birch 62] B. J. Birch. "Forms in Many Variables," Proc. Roy. Soc. Ser. A 265 (1961/1962), 245-263.

[de la Bretèche et al. 07] R. de la Bretèche, T. D. Browning, and U. Derenthal. "On Manin's Conjecture for a Certain Singular Cubic Surface." Ann. Sci. École Norm. Sup. 40:1(2007), 1-50.

[Cohen 93] H. Cohen. A Course in Computational Algebraic Number Theory. Berlin: Springer, 1993.

[Dokchitser 04] T. Dokchitser. "Computing Special Values of Motivic L-Functions." Experiment. Math. 13 (2004), 137149 .

[Elsenhans and Jahnel 06a] A.-S. Elsenhans and J. Jahnel. "The Diophantine Equation $x^{4}+2 y^{4}=z^{4}+4 w^{4}$." Math. Comp. 75 (2006), 935-940.

[Elsenhans and Jahnel 06b] A.-S. Elsenhans and J. Jahnel. "The Asymptotics of Points of Bounded Height on Diagonal Cubic and Quartic Threefolds." In Algorithmic Number Theory, Lecture Notes in Computer Science 4076, pp. 317-332. Springer: Berlin, 2006.

[Elsenhans and Jahnel 09] A.-S. Elsenhans and J. Jahnel. "Experiments with General Cubic Surfaces." In Algebra, Arithmetic and Geometry: Manin Festschrift, Progress in Mathematics 269, pp. 637-654. Boston: Birkhäuser, 2009 .

[Elsenhans and Jahnel 10] A.-S. Elsenhans and J. Jahnel. "On the Smallest Point on a Diagonal Cubic Surface." Exp. Math. 19 (2010), 181-193.

[Franke et al. 89] J. Franke, Y. I. Manin, and Y. Tschinkel. "Rational Points of Bounded Height on Fano Varieties." Invent. Math. 95 (1989), 421-435.

[Hooley 86] C. Hooley. "On Some Topics Connected with Waring's Problem." J. Reine Angew. Math. 369 (1986), $110-153$.

[Peyre 95] E. Peyre. "Hauteurs et mesures de Tamagawa sur les variétés de Fano." Duke Math. J. 79 (1995), 101-218. 
[Peyre 02] E. Peyre. "Points de hauteur bornée et géométrie des variétés (d'après Y. Manin et al.)," Séminaire Bourbaki 2000/2001. Astérisque 282 (2002), 323-344.

[Peyre and Tschinkel 01] E. Peyre and Y. Tschinkel. "Tamagawa Numbers of Diagonal Cubic Surfaces, Numerical Evidence." Math. Comp. 70 (2001), 367-387.
[Silverman 86] J. Silverman. The Arithmetic of Elliptic Curves. Berlin: Springer, 1986.

[Swinnerton-Dyer 05] P. Swinnerton-Dyer. "Counting Points on Cubic Surfaces, II." In Geometric Methods in Algebra and Number Theory, Progr. Math. 235, pp. 303-309. Boston: Birkhäuser, 2005.

Andreas-Stephan Elsenhans, Universität Bayreuth, Mathematisches Institut, Universitätsstrasse 30, D-95447 Bayreuth, Germany (Stephan.Elsenhans@uni-bayreuth.de)

Received January 8, 2010; accepted March 29, 2010. 